\title{
Combined valve surgery with sutureless and rapid-deployment aortic valve replacement: when and why
}

\author{
Stephanie N. Nguyen ${ }^{1}$, Benjamin M. Cohn ${ }^{2}$, Tom C. Nguyen ${ }^{2}$ \\ ${ }^{1}$ Division of Cardiac, Thoracic, and Vascular Surgery, Department of Surgery, New York Presbyterian-Columbia University Medical Center, New \\ York, NY, USA; ${ }^{2}$ Department of Cardiothoracic and Vascular Surgery, University of Texas Health Science Center at Houston, McGovern Medical \\ School, Houston, TX, USA \\ Correspondence to: Tom C. Nguyen, MD. Chief of Cardiac Surgery, Memorial Hermann, Associate Professor, Cardiothoracic Surgery, Director, \\ Minimally Invasive Valve Surgery, University of Texas Health Science Center at Houston, 6400 Fannin Street, Suite 2850, Houston, TX 77030, \\ USA. Email: tom.c.nguyen@gmail.com.
}

Submitted Oct 14, 2019. Accepted for publication Dec 09, 2019.

doi: 10.21037/acs-2019-surd-11

View this article at: http://dx.doi.org/10.21037/acs-2019-surd-11

Given the disruptive growth of transcatheter aortic valve replacement (TAVR) and push towards minimally invasive approaches, cardiac surgeons face a dual challenge: preservation as well as advancement of surgical aortic valve replacement (SAVR). To achieve this, we must leverage new valve technologies in pursuit of an individualized, minimally invasive approach for the aging population with increased morbidities. One method of reducing the risks of surgery includes sutureless or rapid-deployment aortic valve replacement (SURD-AVR), particularly in high-risk patients.

Aortic valve disease is a common issue in an aging population with multiple comorbidities. Many patients also present with concomitant valvular or vessel disease, requiring concomitant procedures; however, significantly prolonged operative times may increase morbidity and mortality in this already high-risk population (1). By avoiding placement and tying of annular sutures, SURD-AVR significantly reduces operative, and more importantly, ischemic times, and may improve outcomes (2-4). In fact, it has been shown to almost halve bypass and cross-clamp times when compared with conventional SAVR (4). Therefore, SURD-AVR should be considered in order to minimize operative times and improve outcomes in high-risk patients in whom a long bypass run would be detrimental, and in those undergoing complex combined procedures.

Clinically significant mitral regurgitation (MR) is often found in conjunction with aortic stenosis (AS); however, whether to address the MR at the time of SAVR remains controversial, with no clear guidelines. While functional
MR may improve with AVR alone, intrinsic MR should be considered for concomitant repair/replacement, as moderate MR is an independent risk factor for long-term mortality in elderly patients undergoing AVR (5). Baran and colleagues report their experience with SURD-AVR in 30 patients undergoing concomitant mitral valve surgery and concluded that it was safe and technically feasible with good hemodynamic performance and low rates of paravalvular leak (PVL); however, patient selection and type of mitral prosthesis are of utmost importance (6).

SURD-AVR is also a feasible option in combined coronary artery bypass grafting (CABG). When compared to traditional SAVR/CABG, SURD-AVR/CABG has been shown to shorten bypass and cross-clamp times (7), which may improve outcomes in those undergoing complex multivessel revascularization. Unlike in SAVR, a high transverse aortotomy, approximately $3.5 \mathrm{~cm}$ distal to the sinotubular junction, is made to accommodate the Perceval Sutureless valve (Sorin/LivaNova Group, Saluggia, Italy) (7), whereas a lower transverse aortotomy may facilitate the Edwards Intuity Elite valve (Edwards Lifesciences, Corp., Irvine, CA USA) (8). Thus, an essential component involves preoperative planning of the location of proximal anastomoses, prior to cannulation.

Another potential indication for SURD-AVR includes patients with a small, calcified aortic annulus. In contrast to TAVR, SURD-AVR enables complete native leaflet excision and comprehensive annular decalcification, in order to optimize valve position and avoid a PVL. For those 
with circumferential annular calcification in whom safe debridement and suture placement are not possible, SURDAVR enables valve deployment under direct visualization and repositioning, as necessary. We recommend performing SURD-AVR as the final step in a combined valve operation, in order to minimize manipulation of the seated valve. SURD-AVR is also useful in patients with a small aortic root $(<21 \mathrm{~mm})$. One study reported excellent hemodynamic performance of SURD-AVR compared to SAVR, with significantly lower rates of patient-prosthetic mismatch (PPM) (9). Therefore, SURD-AVR may reduce complications of PPM while simplifying the operation by potentially avoiding a root enlargement. This is particularly important in elderly, high-risk patients, in whom extended pump runs may be detrimental.

Minimally invasive AVRs are associated with greater patient satisfaction and fewer post-operative complications. With its compact design and easily handling, SURD-AVR facilitates a minimally invasive approach which has been shown to be of comparable quality to a full sternotomy for AVR (10). Despite the technical challenge of working through a smaller incision with long instruments, minimally invasive SURD-AVR obviates the challenging placement and tying of sutures. In experienced hands, it has been shown to reduce bypass and cross-clamp times compared with SAVR via full sternotomy (7). For SURD-AVR, we favor a right anterior mini-thoracotomy approach as we believe it is more ergonomically favorable than a partial sternotomy and provides direct access to the aortic valve. This versatile exposure allows easy access for concomitant intervention, including triple valve surgery. Along with the known durability of surgical tissue valves, ability to ensure optimal valve seating, and decreased operative times, it is evident that this should be one of the preferred approaches.

Despite the efficiency and versatility of SURDAVR, there are important anatomical contraindications to consider. With regards to the aortic root geometry, implantation of the Perceval Sutureless valve (Sorin/ LivaNova Group) is contraindicated in patients with a sinotubular junction to annulus diameter $<1.3$ and in those with an elongated, pear-shaped root. In addition, since the largest available prostheses is limited to an annular size of $27 \mathrm{~mm}$, SURD-AVR is contraindicated in cases of degenerative aortic insufficiency and aneurysmal dilatation, due to risk of PVL and device migration. It should also be avoided in patients with an annulus size $<19 \mathrm{~mm}$, requiring a root enlargement, as patch material confers less stability to anchor the deployed valve. Moreover, there is paucity of data on concomitant valve surgery and SURDAVR, with some cautioning against its technical feasibility under certain circumstances (3). Careful assessment of the aorto-mitral distance (AMD) is critical to avoid prosthesis interference or supra-annular malpositioning during deployment, both of which are lessons learned from prior experience with TAVR.

A minimum AMD of $5-8 \mathrm{~mm}$ is advised for safe deployment of the sutureless aortic valve when there is a mitral prosthesis in place (3). Furthermore, SURDAVR is contraindicated in patients with Sievers type 0 bicuspid aortic valve, bulky calcium not amenable to safe decalcification, or an annular abscess or destruction secondary to infective endocarditis.

In conclusion, SURD-AVR may improve clinical outcomes in high-risk patients undergoing combined valve surgery, owing to a reduction in operative and myocardial ischemic times. It also facilitates comprehensive annular decalcification and leaflet excision, ensuring optimal valve positioning under direct visualization. Though technical challenges and paucity of long-term outcomes exist, we anticipate further evolution of this technology to produce a more durable valve, which can be used to simplify and shorten complex operations in high-risk patients.

\section{Acknowledgments}

None.

\section{Footnote}

Conflicts of Interest: TCN: Edwards LifeSciencesConsultant. SNN and BMC have no conflicts of interest to declare.

Open Access Statement: This is an Open Access article distributed in accordance with the Creative Commons Attribution-NonCommercial-NoDerivs 4.0 International License (CC BY-NC-ND 4.0), which permits the noncommercial replication and distribution of the article with the strict proviso that no changes or edits are made and the original work is properly cited (including links to both the formal publication through the relevant DOI and the license). See: https://creativecommons.org/licenses/by-nc-nd/4.0/.

\section{References}

1. Ranucci M, Frigiola A, Menicanti L, et al. Aortic cross- 
clamp time, new prostheses, and outcome in aortic valve replacement. J Heart Valve Dis 2012;21:732-9.

2. Pollari F, Santarpino G, Dell'Aquila AM, et al. Better short-term outcome by using sutureless valves: a propensity-matched score analysis. Ann Thorac Surg 2014;98:611-6; discussion 616-7.

3. Minh TH, Mazine A, Bouhout I, et al. Expanding the indication for sutureless aortic valve replacement to patients with mitral disease. J Thorac Cardiovasc Surg 2014;148:1354-9.

4. Phan K, Tsai YC, Niranjan N, et al. Sutureless aortic valve replacement: a systematic review and meta-analysis. Ann Cardiothorac Surg 2015;4:100-11.

5. Barreiro CJ, Patel ND, Fitton TP, et al. Aortic valve replacement and concomitant mitral valve regurgitation in the elderly: impact on survival and functional outcome. Circulation 2005;112:I443-I447.

6. Baran C, Durdu MS, Gumus F, et al. Sutureless aortic

Cite this article as: Nguyen SN, Cohn BM, Nguyen TC. Combined valve surgery with sutureless and rapid-deployment aortic valve replacement: when and why. Ann Cardiothorac Surg 2020;9(5):408-410. doi: 10.21037/acs-2019-surd-11 valve replacement with concomitant valvular surgery. J Thorac Cardiovasc Surg 2018;155:2414-22.

7. Flameng W, Herregods MC, Hermans H, et al. Effect of sutureless implantation of the Perceval S aortic valve bioprosthesis on intraoperative and early postoperative outcomes. J Thorac Cardiovasc Surg 2011;142:1453-7.

8. Glauber M, Moten SC, Quaini E, et al. International Expert Consensus on Sutureless and Rapid Deployment Valves in Aortic Valve Replacement Using Minimally Invasive Approaches. Innovations (Phila) 2016;11:165-73.

9. Bonderman D, Graf A, Kammerlander AA, et al. Factors determining patient-prosthesis mismatch after aortic valve replacement--a prospective cohort study. PLoS One 2013;8:e81940.

10. Phan K, Xie A, Di Eusanio M, et al. A meta-analysis of minimally invasive versus conventional sternotomy for aortic valve replacement. Ann Thorac Surg 2014;98:1499-511. 